\title{
Evaluation of DCE-MRI postprocessing techniques to assess metastatic bone marrow in patients with prostate cancer
}

\author{
Nicolas Michoux*, Paolo Simoni, Bertrand Tombal, Frank Peeters, \\ Jean-Pascal Machiels, Frédéric Lecouvet \\ Institut de Recherche Expérimentale et Clinique (IREC), Université Catholique de Louvain, Cliniques Universitaires Saint-Luc, Brussels, Belgium
}

Received 9 June 2011; accepted 7 October 2011

\begin{abstract}
Dynamic contrast-enhanced magnetic resonance imaging was performed in control patients with normal bone marrow and patients with untreated bone metastases of prostate cancer (PCa). Perfusion data were assessed using region of interest-based and pixel-wise current standard postprocessing techniques (signal intensity pattern, increase in signal intensity, upslope, time to peak, extended Kety model, $k$ means clustering). Bone marrow perfusion is significantly increased in bone metastases of PCa compared to normal bone marrow. Pixel-wise kinetic modeling should be recommended to assess tumoral processes affecting bone marrow microcirculation.
\end{abstract}

(C) 2012 Elsevier Inc. All rights reserved.

Keywords: Dynamic contrast-enhanced MRI (DCE-MRI); Prostate cancer (PCa); Bone metastases; Imaging biomarkers; Pharmacokinetics

\section{Introduction}

Current imaging screening for bone metastases of prostate cancer (PCa) including technetium-99m bone scan, targeted $\mathrm{X}$-rays, and magnetic resonance imaging (MRI) are not effective in revealing early tumor changes induced by anticancer drugs [1-3]. Dynamic contrast-enhanced MRI (DCE-MRI) is now recognized as a potential early predictor of response to drug therapy and radiotherapy [4-7]. The usefulness of the technique in various benign and malignant musculoskeletal lesions [8] and its prognostic potential in multiple myeloma [9] have been investigated. Despite these results, quantitative DCE-MRI has never reached routine clinical applications in bone imaging. The main reasons for this might be the need for intravenous contrast administration, the suboptimal effectiveness of low-molecular-weight contrast agents for a precise assessment of the marrow

* Corresponding author. Université Catholique de Louvain, Hôpital Saint-Luc, Radiology-Imaging Unit, Avenue Hippocrate 10, 1200 Brussels, Belgium. Tel.: +32 2 7642551; fax: +32 27705574 .

E-mail address: nicolas.michoux@uclouvain.be (N. Michoux). microstructure $[5,10]$, and the measurement of an arterial input function. The computationally intensive postprocessing to get quantitative estimates of the bone marrow microcirculation (including a precontrast mapping of $\mathrm{T} 1$ or a calibration procedure, the modeling of the contrast agent kinetics, and the implementation of a robust data fitting procedure) is also involved. These requirements often lead to choosing the simplest analysis model over more realistic tracer kinetic models. However, as qualitative or semiquantitative models of bone marrow perfusion have never been formally validated [5], more sophisticated models should not be excluded from the process of model selection.

The aim of the study is to estimate the efficiency of the current standard DCE-MRI postprocessing techniques to assess bone marrow microcirculation. Qualitative analysis of signal intensity vs. time (SI) curves, region of interest (ROI)based semiquantitative analysis, ROI-based kinetic modeling, pixel-based kinetic modeling, and kinetic parameters-based clustering were assessed on the basis of two populations: patients with untreated bone metastases of $\mathrm{PCa}$ and control patients with normal bone marrow. Reference values of bone marrow perfusion, blood volume, and interstitial volume for 
both populations were also provided. These values might represent baseline information for further studies focused on perfusion changes in bone metastases of $\mathrm{PCa}$ during treatment.

\section{Materials and methods}

\subsection{Patients}

Ten patients with bone metastases of PCa and 10 control patients without history of cancer were included in the study and underwent an MRI examination. Patients with bone metastases of PCa had newly diagnosed PCa (mean age, 68 \pm 9 years; range $52-80$ years) referred by the oncology and/or urology department to rule out bone metastases of the spine. In all 10 patients, the MRI examination including DCE-MRI was performed before any drug or radiation therapy was started. Because of the lack of histological proof, a 6-month follow-up by standard MRI survey for bone marrow was used as a gold standard for the confirmation of the malignant nature of the lesions considered as metastases and the stability of bone marrow considered as normal. In this 6month interval, patients with $\mathrm{PCa}$ received hormonal therapy, and bone metastases were proved by any change of size and/or signal of the lesion over this time period (see Table 1). Control patients without history of PCa underwent MRI of the lumbar spine with contrast injection. All patients were referred for a low-back-pain MRI workup by an academic orthopedist. Patients with known disease that could potentially alter bone marrow changes were discarded from the analysis. These were patients with history of cancer even without evidence/history of bone metastases, with previous surgery of the lumbar spine, with chronic anemia (defined as hemoglobin level $<12 \mathrm{~g} \mathrm{ml}^{-1}$ ), or with chronic inflammatory disease (defined as reactive C-protein $>1.5 \mathrm{mg}$ $\mathrm{dL}^{-1}$ and/or white blood cells $>10,000 \mathrm{ml}^{-1}$ ). All laboratory tests were performed during the week before the MRI examination. The final control population consisted of eight patients, three men and five women (mean age, $56 \pm 14$ years; range $39-75$ years) with normal bone marrow according to the above-mentioned criteria. The study was approved by the local ethical committee, and written informed consent was obtained from patients.

\subsection{Magnetic resonance standard imaging}

All MRI examinations were performed on a 1.5-T scanner (Gyroscan NT Intera 1.5 T; Philips Medical Systems, Best, the Netherlands) with the body coil. For each patient, a preliminary standard magnetic resonance examination of the spine was performed using $\mathrm{T} 1$ and proton density with fat saturation (PDFS) sequences (sagittal plane). Transverse sections were then programed on bone metastases identified on their preliminary images to delineate these lesions. Control patients referred for back pain were also examined with transverse T1 and T2 images obtained on the anatomic levels relevant to the clinical question. Bone metastases were defined as an area of low signal intensity on T1-weighted images compared to normal muscle and/or disk and intermediate to high signal intensity on the PDFS images.

\subsection{DCE-MRI examination}

Bone marrow perfusion was assessed with a spoiled twodimensional turbo-Fast Low Angle SHot sequence. A nonsliceselective $90^{\circ}$ preparation pulse was incorporated. Scan parameters were as follows: slice thickness $L=10 \mathrm{~mm}$, Field Of View $=262 \times 400 \mathrm{~mm}$, in-plane voxel size $=1.02 \times 1.56 \mathrm{~mm}$ after reconstruction, flip angle $=15^{\circ}$, single shot, echo time $=1.3$ $\mathrm{ms}$, repetition time $=5.4 \mathrm{~ms}$, a shot duration of $516 \mathrm{~ms}$, and 200 dynamics for a total acquisition time of 1 min $47 \mathrm{~s}$. Patients received $8 \mathrm{ml}$ of Gd-DTPA (Magnevist; Bayer Schering Pharma AG, Berlin, Germany) followed by $20 \mathrm{ml}$ saline flush injected at a rate of $3 \mathrm{ml} / \mathrm{s}$ with an automated injector.

A single transverse slice that included bone metastases or normal bone marrow and the aorta was continuously scanned using the fast-gradient echo sequence after contrast agent injection. This single transverse slice was manually positioned passing through the largest metastasis detected at the level of

Table 1

Patients with PCa bone metastases

\begin{tabular}{|c|c|c|c|c|c|c|}
\hline Patients & $\begin{array}{l}\text { Age } \\
\text { (years) }\end{array}$ & $\begin{array}{l}\text { Gleason } \\
\text { score }\end{array}$ & $\begin{array}{l}\text { PSA plasmatic } \\
\text { level }(\mathrm{ng} / \mathrm{ml})\end{array}$ & Total no. of lesions & $\begin{array}{l}\text { Location of the } \\
\text { largest lesion }^{a}\end{array}$ & $\begin{array}{l}\text { Size of the largest } \\
\text { lesion }^{\mathrm{a}}(\mathrm{mm})\end{array}$ \\
\hline 1 & 80 & 8 & $<0.1$ & $>10$ & L2 & 43 \\
\hline 2 & 67 & 8 & 1470 & $>10$ & L4 & 45 \\
\hline 3 & 55 & 9 & 3 & $>10$ & L4 & 45 \\
\hline 4 & 74 & 6 & 11 & Diffuse infiltration & $\mathrm{L} 2^{\mathrm{b}}$ & 45 \\
\hline 5 & 70 & 6 & 1069 & $>10$ & D7 & 16 \\
\hline 6 & 52 & 7 & 1369 & $>10$ & L4 & 26 \\
\hline 7 & 74 & 7 & 115 & $>10$ & L4 & 23 \\
\hline 8 & 69 & 9 & 202 & 9 & L3 & 39 \\
\hline 9 & 65 & 7 & 3 & $>10$ & L5 & 28 \\
\hline 10 & 56 & 7 & 144 & $>10$ & L1 & 16 \\
\hline
\end{tabular}

PSA, Prostate-Specific Antigen.

a Sample lesion for DCE-MRI

${ }^{\mathrm{b}}$ Vertebral body considered as sample lesion: diffuse infiltration of bone marrow. 
the spine on the basis of sagittal slices. In control patients, the single slice was positioned at level of the upper third of the L3 vertebral body to avoid the basivertebral veins and the cortex of vertebral plates. The upper third of L3 vertebral body was chosen because mechanic degenerative changes of bone marrow are by far less common than in L4 and L5.

\subsection{Image postprocessing}

The software Image J (National Institutes of Health, http://rsbweb.nih.gov/ij/) was used for the segmentation of the ROIs. An experienced musculoskeletal radiologist manually drew one ROI covering the aorta and another ROI in the bone marrow. For bone metastasis, a careful delineation of the lesion on the basis of sagittal and axial images obtained before contrast agent injection was performed. For normal marrow, the entire vertebral body (except for the cortices) on the axial image was included in the ROI. The program reported the ROI for each image of the time series and extracted the SI curves.

\subsection{Semiquantitative parameters}

Bone marrow perfusion was first assessed using three simple geometrical measurements of the signal intensity enhancement [11]. The percentage increase (PI) in signal intensity was calculated as follows:

$\mathrm{PI}=\left[\left(\mathrm{SI}_{\max }-\mathrm{SI}_{\sum \text { pre }}\right) * 100\right] / \mathrm{SI}_{\sum \text { pre }}$

where $\mathrm{SI}_{\Sigma \text { pre }}$ is the mean signal intensity measurement in the ROI before contrast agent arrival and $\mathrm{SI}_{\max }$ is the maximum signal intensity measurement in the ROI during the first pass of the contrast agent. The upslope was calculated by linear regression of the signal intensity on the interval $\left[\mathrm{SI}_{\mathrm{pre}}\right.$; $\mathrm{SI}_{\text {max }}$ ], where $\mathrm{SI}_{\text {pre }}$ is the signal intensity measurement measured just before the contrast agent uptake. The time $T_{\max }$ corresponding to the peak $\mathrm{SI}_{\max }$ was also measured.

\subsection{Kinetic parameters}

According to the procedure described in Ref. [12], SI curves were converted into longitudinal relaxation rate $R_{1}$ vs. time curves. A linear relationship was assumed between $R_{1}$ and contrast agent concentration according to the following equation:

$R_{1}=R_{1 \text { post }}-R_{1 \text { pre }}=r_{1} C$

where $R_{1 \text { post }}$ is the postcontrast longitudinal relaxation rate, $R_{1 \text { pre }}$ the precontrast longitudinal relaxation rate, $r_{1}$ the longitudinal relaxivity of the contrast agent, and $C$ is concentration. In this equation, it is assumed that the relaxivity of the contrast agent is the same in blood and tumors and that the exchanges of water between intravascular, extravascular, and intracellular spaces are fast [13].
The extended Kety model with an individual arterial input function was used $[14,15]$. This model assumes a bidirectional exchange between two compartments, the intravascular space (IVS) and the extravascular extracellular space (EES), as well as a contribution of the tracer in IVS. It can be written as:

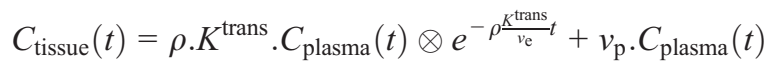

where $\rho\left(\rho=1 \mathrm{~g} \mathrm{ml}^{-1}\right)$ is the tissue density, $C_{\text {plasma }}\left(\mathrm{g} \mathrm{ml}^{-1}\right)$ is the concentration of contrast agent in IVS, $C_{\text {tissue }}\left(\mathrm{g} \mathrm{ml}^{-1}\right)$ is the concentration of contrast agent in the tissue, $K^{\text {trans }}=E \cdot F$ $\left(\mathrm{ml} \mathrm{s}{ }^{-1} \mathrm{~g}^{-1}\right)$ is the product of the extraction fraction and the tissue plasma flow, $v_{\mathrm{e}}(\%)$ is the EES accessible to the contrast agent, $v_{\mathrm{p}}(\%)$ is the fractional plasma volume of the contrast agent, and $\otimes$ denotes the convolution product. The model contains three free parameters to fit, $K^{\text {trans }}, v_{\mathrm{e}}$, and $v_{\mathrm{p}}$. The hematocrit $H_{\mathrm{ct}}$ was fixed to 0.45 for all patients, and the arterial concentration of the tracer in whole blood was converted into the corresponding plasma quantity according to the following formula $C_{\text {plasma }}(t)=C_{\text {blood }}(t) /\left(1-H_{\mathrm{ct}}\right)$.

For each patient, the kinetic model was first fitted to the average $R_{1}$ curve from the ROI. Then, it was fitted on a pixel-by-pixel basis in the entire bone marrow of the vertebra to generate three parametric maps based on $K^{\text {trans }}, v_{\mathrm{e}}$, and $v_{\mathrm{p}}$. To obtain the coefficient $K^{\text {trans }}$ (in $\mathrm{ml} \mathrm{min}^{-1} 100 \mathrm{~g}^{-1}$ ), the coefficient expressed (in $\mathrm{ml} \mathrm{s}^{-1} \mathrm{~g}^{-1}$ ) was multiplied by $60 \mathrm{~s} \mathrm{~min}^{-1}$ and by 100 .

A statistical partitioning of the pixels in bone marrow based on the kinetic parameters was computed. Then, bone metastases margins manually drawn by the radiologist on the basis of T1-weighted imaging on precontrast $\mathrm{T} 1$ axial images were compared to the map generated with the clustering method.

\subsection{Statistical analysis}

Optimization of the fits was achieved with a weighted nonlinear least squares procedure based on the LevenbergMarquardt method combined with a three-dimensional grid of starting parameter values to find the solution corresponding to the true global minimum of the error function [16].

The $k$-means clustering [17] approach, used to partition the pixels in the bone marrow area, was parameterized with a number of final clusters equal to 2 , a preliminary clustering phase on a random $10 \%$ subsample of the pixels and with the L1 distance to calculate the distance between centroid clusters. Pixels belonging to one cluster and showing a distance measure to another cluster less than 0.5 were considered as poorly classified.

Semiquantitative and kinetic parameters were expressed as mean \pm S.D. Patients' age and parameters were compared between both groups with the Wilcoxon rank sum test. A $P$ value of .05 was considered statistically significant. The performance of the various parameters in discriminating the normal bone marrow vs. the metastatic bone marrow was 
studied by using nonparametric receiver operating characteristic (ROC) curves. Cutoff values for PI, upslope, $K^{\text {trans }}$, and $v_{\mathrm{e}}$ as well as the corresponding sensitivity and specificity were computed with exact $95 \%$ confidence intervals based on the $F$ distribution [18]. As the area under the ROC curve (AUC) is a summary measure of the parameter performance, we performed an unpaired statistical comparison of the AUCs. All calculations were done with Matlab (v. 6.5, rel. 13; MathWorks, Natick, MA, USA).

\section{Results}

Patients age did not differ between the controls and the PCa patients $(P=.39)$. Typical SI curves are presented in Fig. 1. In the control patients, an almost flat profile is observed. In bone metastases, the patients exhibited different profiles with as common feature, a fast contrast uptake and either a plateau or a rapid washout, but with an intensity enhancement always much higher to that observed in normal bone marrow. SI curves measured from two ROIs placed in normal and a tumoral area of the same patient with bone metastases revealed similar differences, with a lower and slower intensity enhancement in the normalappearing area of the bone marrow.

Measurements of the semiquantitative and kinetic parameters are summarized in Table 2. Bone metastases showed a significantly higher upslope compared to normal bone marrow $(P=.0002)$ as well as a significantly higher PI $(P=.00005)$. No statistically significant difference was found in the time-to-peak $T_{\max }$ between both groups $(P=.26)$. Bone metastases showed a significantly higher value of $K^{\text {trans }}, v_{\mathrm{e}}$, and $v_{\mathrm{p}}$ compared to normal bone marrow $(P=.0015$, $P=.00004$, and $P=.0048$, respectively).

The best estimated parameters used to predict the bone marrow status were as follows: PI $(\mathrm{AUC}=1.00 \pm 0.00), v_{\mathrm{e}}$ $(\mathrm{AUC}=0.99 \pm 0.03)$, upslope $(\mathrm{AUC}=0.97 \pm 0.05)$, and $K^{\text {trans }}$
Table 2

Mean values $( \pm$ S.D.) of the semiquantitative and kinetic parameters

\begin{tabular}{lllllll}
\hline Patients & Upslope $^{\mathrm{a}}$ & $\mathrm{PI}^{\mathrm{a}}$ & $T_{\max }$ & $K^{\text {transa }}$ & $v_{\mathrm{e}}{ }^{\mathrm{a}}$ & $v_{\mathrm{p}}{ }^{\mathrm{a}}$ \\
\hline PCa & $1.59 \pm 0.57$ & $72 \pm 19$ & $28 \pm 5$ & $57.7 \pm 22.8$ & $12.7 \pm 4.17$ & $1.68 \pm 1.25$ \\
Control & $0.47 \pm 0.27$ & $19 \pm 10$ & $33 \pm 8$ & $18.6 \pm 11.9$ & $4.78 \pm 1.59$ & $1.00 \pm 0.01$
\end{tabular}

Upslope in arbitrary unit $\cdot \mathrm{s}^{-1}$, PI in $\%, T_{\max }$ in $\mathrm{s}, K^{\text {trans }}$ and $k_{\text {ep }}$ in $\mathrm{ml} \mathrm{min}{ }^{-1}$ $100 \mathrm{~g}^{-1}$, and $v_{\mathrm{e}}$, and $v_{\mathrm{p}}$ in $\%$.

${ }^{a}$ Significant differences between metastatic and normal bone marrow (Wilcoxon rank sum test, $P<.05$ ).

(AUC $=0.96 \pm 0.05)$. The most discriminating cutoff values were $\mathrm{PI}<38.02 \%$ (sensitivity, $100 \%$; specificity, 100\%), $v_{\mathrm{e}}<7.41 \%$ (sensitivity, $100 \%$; specificity $90 \%$ ), upslope $<0.92 \mathrm{AU} \cdot \mathrm{s}^{-1}$ (sensitivity, 100\%; specificity, 90\%), and $K^{\text {trans }}<35.73 \mathrm{ml} \mathrm{min}{ }^{-1} 100 \mathrm{~g}^{-1}$ (sensitivity, $100 \%$; specificity, 90\%) for the normal bone marrow. A pairwise comparison of AUC of ROC curves did not yield any statistically significant differences. As a result, it was not possible to distinguish the above parameters according to their performance.

Parametric maps are illustrated in Fig. 2. In normal bone marrow, a homogeneous distribution of low $K^{\text {trans }}, v_{\mathrm{e}}$, and $v_{\mathrm{p}}$ values was typically observed. In bone metastases, parametric maps vary among patients. Distributions of $K^{\text {trans }}, v_{\mathrm{e}}$, and $v_{\mathrm{p}}$ values are heterogeneous, revealing several functionally different areas in the bone marrow.

Clustering based on $K^{\text {trans }}$ and $v_{\mathrm{e}}$ provided maps with the lower number of pixels poorly classified compared to other combinations of two or three parameters including $v_{\mathrm{p}}$. Examples of clustering maps are presented in Fig. 3. In the control patients, the partitioning typically results in a single large area that is consistent with a manual delineation covering the entire bone marrow of the vertebra in the axial plane. In bone metastases, an overall good agreement was observed between the area of the lesion delineated manually and the area identified by the partitioning as an area where
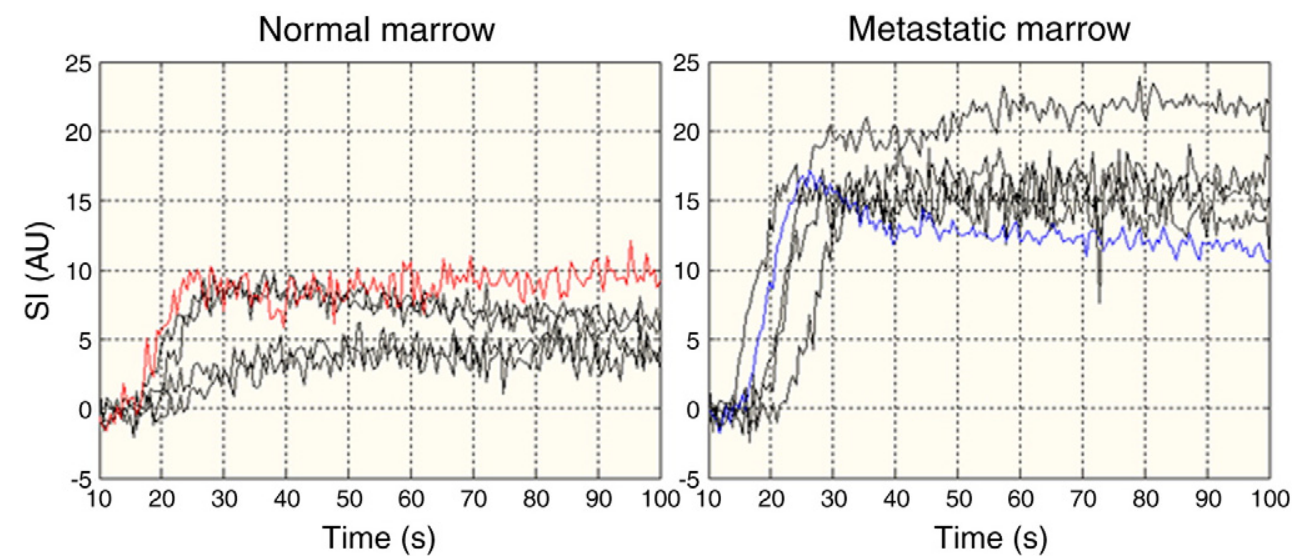

Fig. 1. Example of observed SI curves. The mean signal intensity before the contrast agent injection has been recentered on zero for a better comparison. Normal bone marrow: the two lower black curves correspond to male patients, while the two upper black curves correspond to female patients. The red curve corresponds to the area of normal bone marrow next to the lesion in a metastatic patient (Fig. 2C, nonenhancing area). Untreated bone metastases: the four black curves demonstrate a higher and faster enhancement. The blue curve corresponds to the tumoral area in the second metastatic patient (Fig. 2C, enhancing area). 
$200 \mathrm{ml} / \mathrm{min} / 100 \mathrm{~g}$

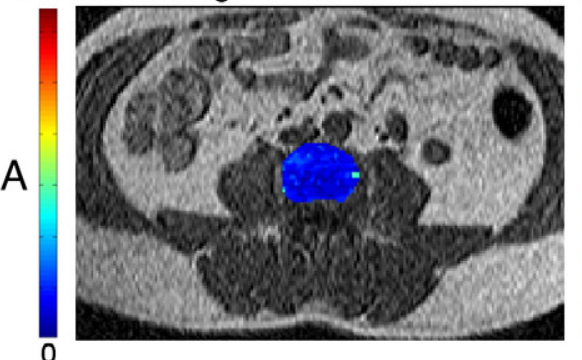

B

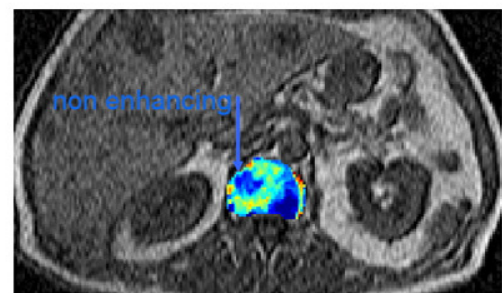

C

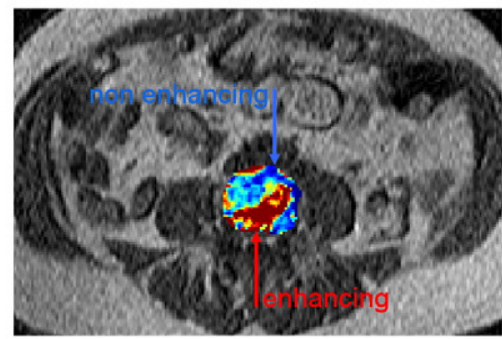

D

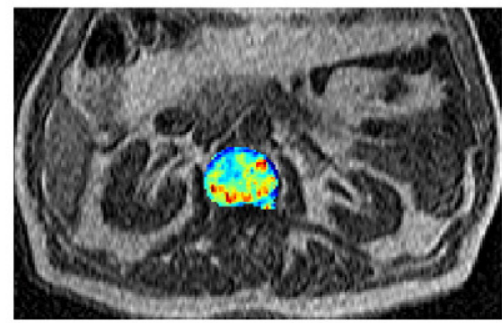

$\mathrm{K}^{\text {trans }}$
$70 \%$
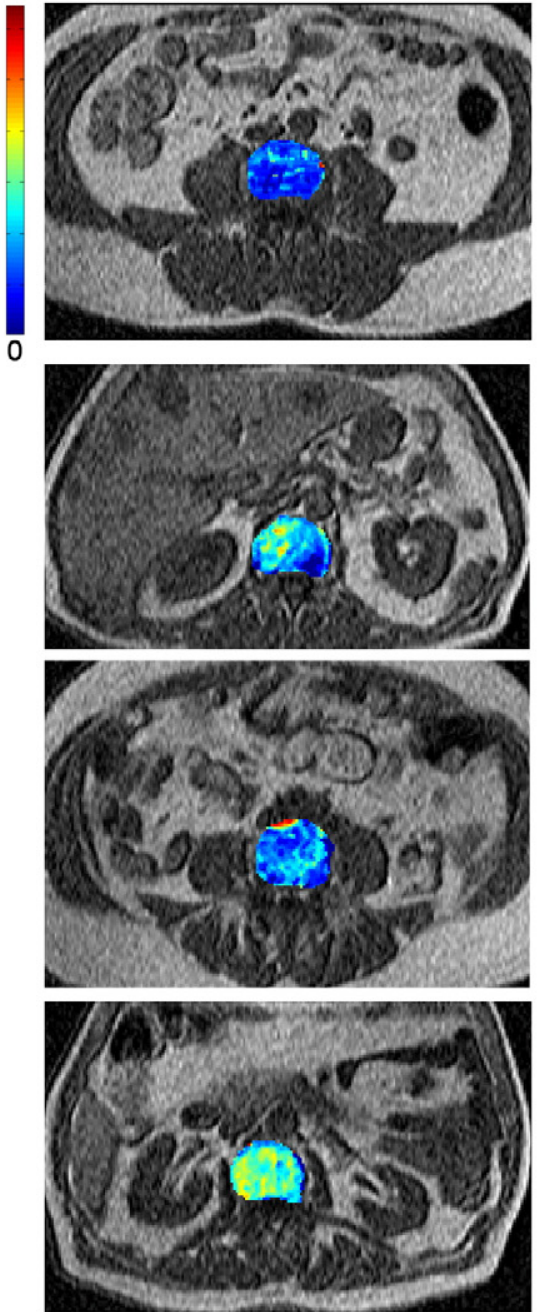

$v_{e}$
$15 \%$
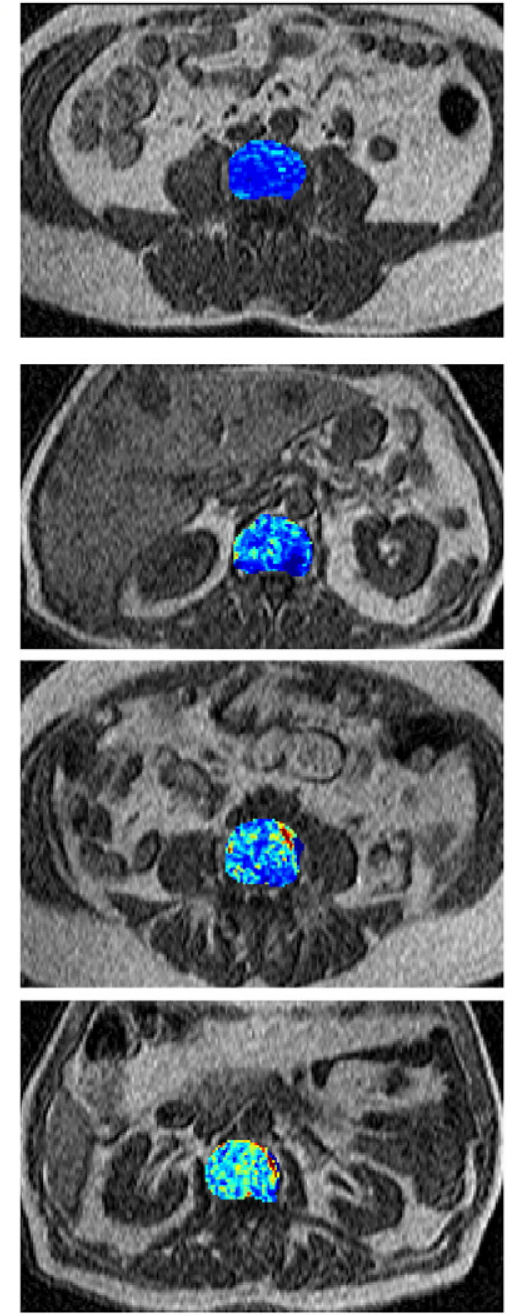

$v_{p}$

Fig. 2. Parametric maps of bone marrow perfusion. The brighter the color code, the higher the value of the functional parameter. (A) A control patient is shown. Maps do not show any local difference. Dark areas representing hypoperfused areas with both small vascular and interstitial volumes are small, sparse, colocalized from one map to the other, and witnesses for an overall low perfusion of the marrow. (B-D) Patients with untreated bone metastases are shown. High values of the transfer constant $K^{\text {trans }}$, interstitial volume $v_{\mathrm{e}}$, and plasma volume $v_{\mathrm{p}}$ are observed. Maps are heterogeneous. (B) One observes that nonenhancing areas cannot be colocalized from one map to the other, which witnesses for the complex characteristics of the metastatic marrow microstructure. (C) Two functionally different areas are present: the nonenhancing area with low perfusion/permeability and moderate interstitial and vascular volumes appearing as normal bone marrow, and the enhancing area with high perfusion/permeability and moderate interstitial and vascular volumes appearing as metastatic marrow.

$K^{\text {trans }}$ values are higher. Substantial differences between both methods of delineation can also be observed.

\section{Discussion}

The main results of the study are as follows. ROI-based semiquantitative and quantitative methodologies provide clear cutoff values for the perfusion parameters, from which bone metastases of PCa can be distinguished from normal bone marrow. Both methodologies show that the perfusion in normal bone marrow is lower compared to that in bone metastases of PCa. Parametric maps based on $K^{\text {trans }}, v_{\mathrm{e}}$, and $v_{\mathrm{p}}$ allow for the characterization of the heterogeneity of bone metastasis. Clustering analysis allows for the segmentation of the lesion based on its measured functional characteristics.

\subsection{Qualitative, semiquantitative, and quantitative methodology}

Qualitative analysis showed that SI curves in patients with bone metastasis are mainly characterized by a marked intensity enhancement. However, various patterns for the washout phase can be observed and are difficult to classify. Analysis of SI patterns has been applied with success to the detection and evaluation of breast cancer [19]. Chen and Shih [11] proposed a classification, more subjective than strict, of SI patterns in patients with compression fracture or 

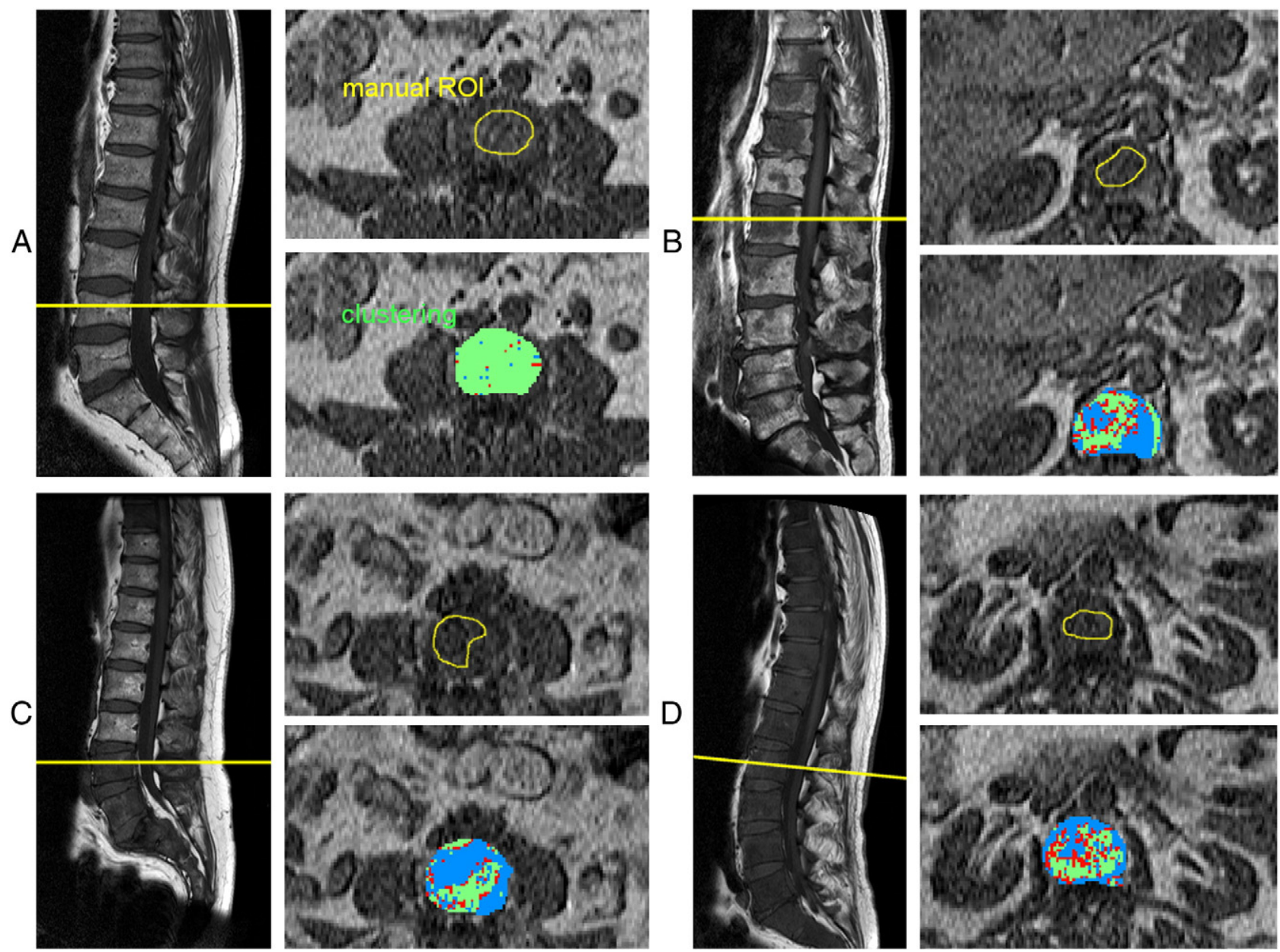

Fig. 3. Sagittal T1-weighted images showing the transverse plan (yellow line) selected for the perfusion sequence, manual ROIs, and clustering maps superimposed on the first image of the perfusion sequence before contrast agent injection. The statistical partitioning of the pixels is estimated from the functional parameters $K^{\text {trans }}$ and $v_{\mathrm{e}}$. The pixel group with the lower mean $K^{\text {trans }}$ value is coded in blue, while the pixels group with the higher mean $K^{\text {trans }}$ value is coded in green. Pixels in red correspond to a poor classification result. Same patients as in Fig. 2 are presented. (A) A control patient is shown. The clustering results in one type of tissue only. (B-D) Patients with untreated bone metastases are shown. The clustering reveals two distinct areas, especially in patients B and C, suggesting two distinct types of tissue in the bone marrow. Pixels with a poor classification result are mainly localized on the border of both areas.

metastatic vertebral lesions. Our results suggest that the analysis of SI patterns remains not very specific and is mainly useful for detecting an abrupt change in the bone marrow perfusion.

Values reported in the literature for semiquantitative parameters vary greatly $[11,20,21]$. In our study, PI ranged between $10 \%$ and $29 \%$ for the control patients and between $53 \%$ and $91 \%$ for patients with PCa. This result is consistent with Baur et al. [22], who suggested that a PI less $40 \%$ is observed in normal bone marrow of adults older than 30 years. Likewise, our values for the upslope ranged between 0.20 and 0.74 for the control patients and between 1.02 and 2.16 for patients with $\mathrm{PCa}$, which is consistent with the results of Montazel et al. [20] in patients with newly diagnosed hematologic malignancies. In another study [23], the same team showed that PI and upslope increased significantly with the degree of bone marrow involvement.

Parameters $K^{\text {trans }}, v_{\mathrm{e}}$, and $v_{\mathrm{p}}$ were significantly lower in normal bone marrow than in bone metastases. Few data in the literature are available for comparison. Hillengass et al. [9], using the model of Brix et al. [24], reported that high values of parameters amplitude $A$ (similar to our parameter $\mathrm{PI}$ ) and exchange rate $k_{\mathrm{ep}}$ indicated diffuse or focal infiltration of the bone marrow. Our study yielded $k_{\text {ep }}$ values $375 \pm 173 \mathrm{ml} \mathrm{min}^{-1} 100 \mathrm{~g}^{-1}$ in the control patients and $478 \pm 214 \mathrm{ml} \mathrm{min}^{-1} 100 \mathrm{~g}^{-1}$ in patients with bone metastasis, similarly to the findings of Hillengass et al. (where $k_{\text {ep }}$ ranged from 0 to $798 \mathrm{ml} \mathrm{min}{ }^{-1} 100 \mathrm{~g}^{-1}$ ). We did not observe any significant difference in $k_{\text {ep }}$ values between the controls and the metastatic patients, but the role of a particular kinetic parameter as diagnostic variable may depend on the studied pathology.

\subsection{Comparison of the methodologies}

Similar performance, as well as substantial correlations between the parameters ( $r \geq 0.7$, data not shown), was found between semiquantitative and quantitative methodology. 
Semiquantitative parameters depend on the definition of the interval $\left[\mathrm{SI}_{\text {pre}} ; \mathrm{SI}_{\max }\right]$. Interval limits are sensitive to signal-to-noise ratio and may result from a subjective choice when the contrast agent uptake and washout are both slow, which is the case in normal bone marrow. Moreover, semiquantitative parameters are well known to be dependent on MRI protocols (magnetic field, coils, and sequence parameters) and baseline $\mathrm{T} 1$ relaxation time of the tissue before contrast injection. Recently, a series of curves features (defined from areas measurement under SI curves normalized to account for both baseline signal intensity and signal intensity $3 \mathrm{~min}$ after contrast agent injection) have been presented as insensitive to $\mathrm{T} 1$ value and protocol parameters [25]. However, first assessment in patients with osteosarcoma showed only a weak correlation with the transfer constant $K^{\text {trans }}$

Kinetic modeling requires an accurate determination of the arterial input function (AIF) [26] and baseline T1 value. Kinetic parameters have a clear physiological meaning, though simplified regarding actual transport mechanisms $[27,28]$. The extended Kety model has been reported as a compromise between the Kety model, neglecting the vascular contribution of the contrast agent, and the more physiological (and more complex) St Lawrence and Lee model, giving more accurate results but being also less precise because of the interdependency of the parameters and their sensitivity to initial values [29]. A recent study [30] suggested that kinetic models should explicitly incorporate a fraction of fat (as an additional parameter to be fitted or assume not fat because of using a perfect fact saturation radio frequency pulse) to get unbiased measurements of the bone marrow perfusion.

\subsection{Pixel-wise quantitative methodology}

Heterogeneity of tumoral perfusion may bias the interpretation of ROI-based parameters [31]. Our results confirm that fact, showing that the spatial distribution of $K^{\text {trans }}, v_{\mathrm{e}}$, and $v_{\mathrm{p}}$ values is heterogeneous in bone metastases, while it is not in normal bone marrow. Therefore, the mean perfusion parameters are significant in normal bone marrow only. Beside, pixel-based methodologies may allow for the monitoring of the effects of vascular-targeting agents on each perfusion parameter in the area of the lesion, in its immediate vicinity, and in more distant locations [32]. To test this hypothesis, we performed a short study on 10 PCa patients with known lumbar metastases scheduled to receive hormonal therapy or Taxotere (Sanofi-Aventis) therapy. These patients were imaged within 1 week before and 7 and 90 days after initial treatment. Our results showed that $K^{\text {trans }}$, $v_{\mathrm{e}}$, and $v_{\mathrm{p}}$ maps were sensitive to treatment changes and allowed for the monitoring of the complex changes occurring in the perfusion in response to therapy [33].

Clustering based on the kinetic parameters allows for the identification of regions with similar functional characteristics within the bone marrow. Our results show that in the control patients, the bone marrow cannot be partitioned. In patients with bone metastasis, the marrow of the involved vertebra is partitioned into two distinct regions: the lesion (identified a posteriori by comparison with a manual delineation), where $K^{\text {trans }}$ is high, and the normal-appearing perilesional tissues, where $K^{\text {trans }}$ is low. Interestingly, the clustering maps result in a more detailed segmentation of the lesion compared to the margins of the bone metastases drawn on the basis of T1-weighted imaging. We can speculate that DCE-MRI may reveal areas of subtle bone marrow infiltration at margins of the lesion in territories appearing normal on standard T1-weighted sequences as previously demonstrated in myeloma [22]. Similar statistical approaches of DCE-MRI data already proved to be useful in detecting and delineating the tumor (which, by extension, allows for the quantitative measurement of the extension or the shrinkage of the tumor between two examinations) and grading its aggressiveness [34].

\subsection{Limitations}

The population we enrolled in this study was limited to 10 patients because we focused our analysis on newly diagnosed untreated bone metastases of $\mathrm{PCa}$ to provide baseline values of perfusion, without biases induced by previous drug therapy. The control population included five women. Several previous articles reported a significant higher perfusion of bone marrow in younger patients, especially in women in fertile age [22]. This may, however, be considered as particularly suitable to determine a series of cutoff values of perfusion between normal bone marrow and bone metastases of $\mathrm{PCa}$.

For each patient, we only studied the larger metastasis in order to obtain the largest ROI and the highest signal-tonoise ratio to fit the curve of enhancement. We deliberately avoided to simultaneously image more than one metastases, which would have been possible by orientating the single slice on sagittal plane, parallel to the spine. An axial plane focuses the analysis to single-bone metastases but also provides a measurement of the AIF that is less prone to flow and pulsation artifacts as well as to partial volume effects. As bone metastases represent a heterogeneous population of malignant lesions, we are aware that the choice of a lesion as a sample to monitor the response to drug therapy would be purely arbitrary in clinical settings.

In conclusion, pixel-wise kinetic modeling should constitute the reference methodology to assess bone marrow microcirculation. The information provided by kinetic parameters regarding the tumoral processes affecting the bone marrow microcirculation justifies the additional cost in data postprocessing. Especially, the measurement of an arterial input function is no more a limiting factor since alternative methods of AIF generation are currently investigated [35] and may offer the possibility of acquiring images with a higher spatial resolution and/or signal-to-noise ratio, as well as performing accurate kinetic modeling of DCE-MRI data in the whole spine. Future investigations 
should focus on the relevance of $K^{\text {trans }}, v_{\mathrm{e}}$, and $v_{\mathrm{p}}$ maps as imaging biomarkers in the treatment monitoring and follow-up of bone metastases of PCa treated by antiangiogenetic/cytotoxic drugs.

\section{References}

[1] Chi KN, Bjartell A, Dearnaley D, et al. Castration-resistant prostate cancer: from new pathophysiology to new treatment targets. Eur Urol 2009;56:594-605.

[2] Biswal S, Resnick DL, Hoffman JM, Gambhir SS. Molecular imaging: integration of molecular imaging into the musculoskeletal imaging practice. Radiology 2007;244:651-71.

[3] Bäuerle $\mathrm{T}$, Bartlinga $\mathrm{S}$, Berger $\mathrm{M}$, et al. Imaging anti-angiogenic treatment response with DCE-VCT, DCE-MRI and DWI in an animal model of breast cancer bone metastasis. Eur J Radiology 2010;73: $280-7$.

[4] Taylor JS, Reddick WE. Evolution from empirical dynamic contrastenhanced magnetic resonance imaging to pharmacokinetic MRI. Adv Drug Deliv Rev 2000;41:91-110.

[5] Daldrup-Link HE, Tobias Henning T, Link TM. MR imaging of therapy-induced changes of bone marrow. Eur Radiol 2007;17:743-61.

[6] Leach MO, Brindle KM, Evelhoch JL, et al. The assessment of antiangiogenic and antivascular therapies in early-stage clinical trials using magnetic resonance imaging: issues and recommendations. Br J Cancer 2005;92:1599-610.

[7] O'Connor JP, Jackson A, Parker GJ, Jayson GC. DCE-MRI biomarkers in the clinical evaluation of antiangiogenic and vascular disrupting agents. Br J Cancer 2007;96:189-95.

[8] Hawighorst H, Libicher M, Knopp MV, Moehler T, Kauffmann GW, van Kaick G. Evaluation of angiogenesis and perfusion of bone marrow lesions: role of semiquantitative and quantitative dynamic MRI. J Magn Reson Imaging 1999;10:286-94.

[9] Hillengass J, Wasser K, Delorme S, et al. Lumbar bone marrow microcirculation measurements from dynamic contrast-enhanced magnetic resonance imaging is a predictor of event-free survival in progressive multiple myeloma. Clin Cancer Res 2007;13:475-81.

[10] Dafni H, Kim S-J, Bankson JA, Sankaranarayanapillai M, Ronen SM. Macromolecular dynamic contrast-enhanced (DCE)-MRI detects reduced vascular permeability in a prostate cancer bone metastasis model following anti-platelet-derived growth factor receptor (PDGFR) therapy, indicating a drop in vascular endothelial growth factor receptor (VEGFR) activation. Magn Reson Med 2008;60:822-33.

[11] Chen W-T, Shih TT. Correlation between the bone marrow blood perfusion and lipid water content on the lumbar spine in female subjects. J Magn Reson Imaging 2006;24:176-81.

[12] Materne R, Smith AM, Peeters F, et al. Assessment of hepatic perfusion parameters with dynamic MRI. Magn Reson Med 2002;47: $135-42$.

[13] Landis CS, Li X, Telang FW, et al. Determination of the MRI contrast agent concentration time course in vivo following bolus injection: effect of equilibrium transcytolemmal water exchange. Magn Reson Med 2000;44:563-74.

[14] Larsson HB, Fritz-Hansen T, Rostrup E, Sondergaard L, Ring P, Henriksen O. Myocardial perfusion modeling using MRI. Magn Reson Med 1996;35:716-26.

[15] Tofts PS, Brix G, Buckley DL, et al. Estimating kinetic parameters from dynamic contrast-enhanced T(1)-weighted MRI of a diffusable tracer: standardized quantities and symbols. J Magn Reson Imaging 1999;10:223-32.

[16] Ahearn TS, Staff RT, Redpath TW, Semple SIK. The use of the Levenberg-Marquardt curve-fitting algorithm in pharmacokinetic modelling of DCE-MRI data. Phys Med Biol 2005;50:85-92.
[17] MacQueen JB. Some methods for classification and analysis of multivariate observations. Proc. 5th Berkeley Symposium on Mathematical Statistics and Probability, Berkeley, University of California Press, 1, 1967. p. 281-97.

[18] DeLong ER, DeLong DM, Clarke-Pearson DL. Comparing the areas under two or more correlated receiver operating characteristic curves: a non parametric approach. Biometrics 1988;44:837-45.

[19] Kuhl C. The current status of breast MR imaging. Part I. Choice of technique, image interpretation, diagnostic accuracy, and transfer to clinical practice. Radiology 2007;244:356-78.

[20] Montazel J-L, Divine M, Lepage E, Kobeiter H, Breil S, Rahmouni A. Normal spinal bone marrow in adults: dynamic gadolinium-enhanced MR imaging. Radiology 2003;229:703-9.

[21] Griffith JF, Yeung DKW, Antonio GE, et al. Vertebral bone mineral density, marrow perfusion, and fat content in healthy men and men with osteoporosis: dynamic contrast-enhanced MR imaging and MR spectroscopy. Radiology 2005;236:945-51.

[22] Baur A, Stäbler A, Bartl R, Lamerz R, Scheidler J, Reiser M. MRI gadolinium enhancement of bone marrow: age-related changes in normals and in diffuse neoplastic infiltration. Skeletal Radiol 1997;26: $414-8$.

[23] Rahmouni A, Montazel J-L, Divine M, et al. Bone marrow with diffuse tumor infiltration in patients with lymphoproliferative diseases: dynamic gadolinium-enhanced MR imaging. Radiology 2003;229: $710-7$.

[24] Brix G, Semmler W, Port R, et al. Pharmacokinetic parameters in CNS Gd DTPA enhanced MRI. J Comput Assist Tomogr 1991;15:621-727.

[25] Guo J-Y, Reddick WE. DCE-MRI pixel-by-pixel quantitative curve pattern analysis and its application to osteosarcoma. J Magn Reson Imaging 2009;30:177-84.

[26] Yang C, Karczmar GS, Medved M, Oto A, Zamora M, Stadler WM. Reproducibility assessment of a multiple reference tissue method for quantitative dynamic contrast enhanced-MRI analysis. Magn Reson Med 2009:61:851-9.

[27] Jain RK. Transport of molecules in the tumor interstitium: a review. Cancer Res 1987;47:3039-51.

[28] Condon MS. The role of the stromal microenvironment in prostate cancer. Seminars in Cancer Biology 2005;15:132-7.

[29] Buckley DL, Roberts C, Parker GJ, Logue JP, Hutchinson CE. Prostate cancer: evaluation of vascular characteristics with dynamic contrastenhanced T1-weighted MR imaging-initial experience. Radiology 2004;233:709-15.

[30] Biffar A, Sourbron S, Schmidt G, et al. Measurement of perfusion and permeability from dynamic contrast-enhanced MRI in normal and pathological vertebral bone marrow. Magn Reson Med 2010;64: $115-24$

[31] Peck KK, Slater G, Wang X, et al. Applications of dynamic contrastenhanced MRI in assessment of spinal bone marrow. Proceedings of the 17th Annual Meeting of ISMRM, Honolulu, Hawaii, USA, 2009. p. 4224. [abstract].

[32] Montemurro F, Russo F, Martincich L, et al. Dynamic contrast enhanced magnetic resonance imaging in monitoring bone metastases in breast cancer patients receiving bisphosphonates and endocrine therapy. Acta Radiol 2004;45:71-4.

[33] Michoux N, Tombal B, Machiels JP, Lecouvet F. Follow-up of parametric maps of the tumoral perfusion in patients with treated bone metastases of prostate cancer. Proceedings of the 18th Annual Meeting of ISMRM, Stockholm, Sweden, 2010. p. 3211. [abstract].

[34] Stoutjesdijk MJ, Veltman J, Huisman H, et al. Automated analysis of contrast enhancement in breast MRI lesions using mean shift clustering for ROI selection. J Magn Reson Imaging 2007;26:606-14.

[35] Yankeelov T, Luci J, Lepage M, et al. Quantitative pharmacokinetic analysis of DCE-MRI data without an arterial input function: a reference region model. Magn Reson Imaging 2005;25:519-29. 